\title{
Permanent Childhood Hearing Loss in Infants with Hypoxic Ischaemic Encephalopathy: Incidence and Risk Factors
}

\author{
Ebtehal Hamed ${ }^{1}$, Nazakat Merchant ${ }^{1}$ and Alpana M Kulkarni ${ }^{2 *}$ \\ ${ }^{1}$ West Herts Hospital NHS Trust, Neonatal Unit, Watford General Hospital, Vicarage Road, Watford, WD18 OHB, UK \\ ${ }^{2}$ Hertfordshire Community NHS Trust, Paediatric Audiology, Peace Children's Centre, Peace Prospect, Watford, WD17 3EW, UK
}

*Corresponding author: Alpana M Kulkarni, Consultant in Audiovestibular Medicine, Peace Children's Centre, Peace Prospect, Watford, WD17 3EW, UK

\section{Abstract}

Objective: To study the incidence of permanent hearing loss in infants with hypoxic ischaemic encephalopathy (HIE) and the risk factors associated with hearing loss.

Study design: A single centre retrospective cohort study was completed in all term and near term infants with HIE over 9 years (2010-18). Newborn screening with one/two stage protocol was carried out initially (automated otoacoustic emissions only or with automated auditory brainstem testing if baby in NICU for more than 48 hours) of all babies with HIE. Babies with Stage 2 and 3 HIE were cooled. Those who failed this protocol had diagnostic auditory brainstem testing (ABR) which identified the hearing loss as sensorineural or auditory neuropathy spectrum disorder. Aetiological investigations were done to rule out other causes of sensorineural hearing loss including MRI IAMS, cytomegalovirus and Connexin 26 testing.

Results: Ninety-nine babies were eligible and 8 died. Ten out of 91 babies failed the newborn screen. Of the 10, 8 (8.8\%) had permanent hearing loss. Six out of 8 babies had bilateral sensorineural hearing loss, 1 had unilateral sensorineural hearing loss and 1 had unilateral auditory neuropathy spectrum disorder. Permanent hearing loss was associated with low Apgar score at 1 and $5 \mathrm{~min}$ (CI 0.5, 3.9, p=0.01), persistent pulmonary hypertension ( $p=0.0002$, CI- $0.5,-0.2)$ and loop diuretics (CI-0.4, $-0.1, p=0.0002)$. Permanent hearing loss was found in all grades of HIE.

Conclusion: This study adds to the previous studies indicating that there is high incidence of permanent hearing loss seen in term and near term babies with HIE (cooled as well as not cooled). Further studies with larger samples are needed to study the risk factors in this cohort and compare the incidence of hearing loss in cooled versus not cooled babies.

What's new: This study confirms that permanent hearing loss of all degree and severity can occur in all grades of hypoxic ischaemic encephalopathy. It indicates that auditory neuropathy spectrum disorder can develop secondary to HIE. Association between low Apgar score at 1 and $5 \mathrm{~min}$, PPHN, loop diuretics and development of permanent hearing loss is significant and needs to be explored further.

Keywords: Permanent hearing loss; Hypoxic ischemic encephalopathy

Abbreviations: PCHI-Permanent childhood hearing impairment; SNHL-Sensorineural hearing loss; HIE-Hypoxic Ischaemic Encephalopathy; NHSP-Newborn hearing screening programme; AOAE-Automated otoacoustic emissions; AABR (Automated auditory brainstem testing); BSA (British society of audiology); dbHL (decibel hearing loss); NICU- Neonatal Intensive Care Unit

\section{Introduction}

Neonatal hypoxic-ischaemic encephalopathy (HIE) is one of the most common causes of cerebral palsy (CP) and other severe neurological deficits in children. Recent trials have shown that thera peutic hypothermia is neuroprotective and did not significantly reduce the combined rate of death or severe disability but resulted in improved neurologic outcome in survivors [1]. Hearing loss is 
a common anomaly presenting at birth (1/500-1/1000) [2]. It is well-documented that infants with HIE have an increased risk for developing sensorineural hearing loss (SNHL) [3]. The severity and duration of the asphyxia might influence whether the impairment is transient or persistent [4]. Recent study has documented that there is high incidence of permanent hearing loss (10.1\%) in cooled babies and high gentamicin was a risk factor [5]. In this and another recent study babies enrolled had moderate to severe HIE [6]. One study recently has shown that hearing screening can be abnormal even in mild HIE [7].

\section{Objectives}

The aim of this study is to assess the incidence of permanent hearing loss in infants with HIE and to investigate contributing risk factors that may increase the risk of acquiring permanent childhood hearing impairment (PCHI).

\section{Patients and Methods}

Retrospective targeted data of all infants with HIE (mild, moderate and severe) were collated from the neonatal unit of a single busy district general hospital over 9 years (2010-2018). Deaths and babies with temporary hearing impairment due to middle ear effusion were excluded. Only infants with term and near term gestation were included. All infants with stage 2 or 3 (moderate and severe) HIE were cooled. Detailed demographic and clinical data were collated which included gestation, birthweight, Apgars, HIE staging, mode of delivery, antenatal and postnatal complications such as chorioamnionitis, persistent pulmonary hypertension, mode and duration of ventilation. Detailed information on medications used especially anticonvulsants, gentamicin and diuretics along with duration and serum levels if known were looked at.

All babies had newborn hearing screening programme (NHSP) using the protocol consisting of Automatic Otoacoustic Emissions (AOES) only or AOES followed by Automated Auditory Brainstem testing (AABR); (the latter if they stayed in neonatal intensive care unit for more than 48 hours) [8]. Those who failed this protocol had a diagnostic Auditory Brainstem Testing as per the NHSP and BSA (British Society of Audiology) recommended standards [9]. A further age appropriate behavioural audiological testing of all the babies who needed follow up was done as per the BSA recommended procedures [10]. All babies with PCHI were offered aetiological investigations as per BAAP (British Association of Audiovestibular Physicians) guidelines for sensorineural hearing loss [11] and early intervention was offered as per the NHSP protocol. The degree of hearing loss was based on BSA recommendation (Table 1).

Table 1

\begin{tabular}{|c|c|}
\hline Descriptor & Average Hearing Threshold Levels (dbHL) \\
\hline Mild hearing loss & $21-40$ \\
\hline Moderate hearing loss & $41-70$ \\
\hline Severe hearing loss & $71-95$ \\
\hline Profound hearing loss & In excess of 95 \\
\hline
\end{tabular}

Results were collated and analysed using STATA 12 and Microsoft excel. Logistic regression analysis and test was used, $p$ value of less than 0.05 was used as the cut off for significance. The study had local research ethics approval.

\section{Results}

Ninety-nine patients were assessed for eligibility in this study who were admitted with HIE over 9 years. Ninety-one infants with HIE were included and 8 were excluded due to death. Ten out of 91 infants failed the hearing screen, 2 patients had temporary hearing loss and were excluded and 8 (8.8\%) had PCHI (Figure 1).

The mean gestational age (GA) was $39.4 \pm$ SD 1.52 weeks in the group of HIE without PCHI and $40 \pm 1.07$ weeks in the group of HIE with PCHI. Most of the baseline perinatal characteristics were similar between both groups of HIE without PCHI and HIE with PCHI. However, the mean Apgar score at $1 \mathrm{~min}$ was 3.1 in the group without PCHI and 0.9 in the group with PCHI (CI 0.5, 3.9, p=0.01) and at $5 \mathrm{~min}$ was 5.4 in the group without $\mathrm{PCHI}$ and 2.9 in the group with PCHI (CI 0.6, 4.4, p=0.01) See (Table 2). There was a significant risk of PCHI in patient with HIE who had persistent pulmonary hypertension (PPHN) ( $p=0.0002, \mathrm{CI}-0.5,-0.2)$ and significant risk of PCHI in those who had loop diuretics during their stay (CI -0.4 , $-0.1, p=0.0002$ ) (Table 2).

Table 2: Baseline perinatal characteristics.

\begin{tabular}{|c|c|c|c|}
\hline 2010-2018 & HIE without PCHI & HIE with PCHI & P (CI) \\
\hline Mean Gestation weeks (SD) & $39.42(1.52)$ & $40(1.07)$ & $0.3(-1.7,0.5)$ \\
\hline Mean birth weight gm (SD) & $3362.64(620.0)$ & $3119.5(461.4)$ & $0.3(-204.8,691.1)$ \\
\hline Sex M: F & $44: 39$ & $1: 01$ & $0.9(-0.4,0.3)$ \\
\hline & Antenatal and delivery risk factors & $0.34(-0.5,0.2)$ \\
\hline CTG suboptimal & 62 & 7 & $0.85(-0.4,0.3)$ \\
\hline FHR bradycardia & 14 & 2 & \\
\hline
\end{tabular}




\begin{tabular}{|c|c|c|c|}
\hline Prelabour CS & 12 & 3 & $0.3(-0.41 .5)$ \\
\hline Inlabour CS & 38 & 3 & \\
\hline SVD & 17 & 1 & \\
\hline Instrumental & 16 & 1 & \\
\hline \multicolumn{4}{|l|}{ Apgar score (mean) } \\
\hline $1 \mathrm{~min}$ & 3.1 & 0.9 & $0.01(0.5,3.9)$ \\
\hline $5 \mathrm{~min}$ & 5.4 & 2.9 & $0.01(0.6,4.4)$ \\
\hline $10 \mathrm{~min}$ & 6.8 & 5.1 & $0.1(-0.3,3.6)$ \\
\hline \multicolumn{4}{|c|}{ Postnatal risk factors } \\
\hline HIE stages & $36: 37: 10$ & 3:03:02 & $0.5(-0.7,0.3)$ \\
\hline Ventilator Days (mean) & 1.1 & 2.6 & $0.07(-3.2,0.1)$ \\
\hline Meconium & 34 & 6 & $0.06(-0.7,0.2)$ \\
\hline PPHN & 3 & 3 & $0.0002(-0.5,-0.2)$ \\
\hline Seizures & 26 & 4 & $0.29(-0.5,0.2)$ \\
\hline Aminoglycoside & 68 & 8 & $0.19(-0.5,0.1)$ \\
\hline High level of aminoglycoside & 10 & 1 & $0.97(-0.5,0.1)$ \\
\hline Loop diuretics & 1 & 2 & $0.0002(-0.4,-0.1)$ \\
\hline Abnormal newborn hearing screen & 2 & 8 & $0.0001(-1.1,-0.9)$ \\
\hline
\end{tabular}

CTG-Cardiotocography, FHR- fetal heart rate, CS- Caesarean section, SVD- spontaneous vaginal delivery, PPHN- persistent pulmonary hypertension of newborn).

\section{Enrollment}

6 out of the 8 patients with PCHI had bilateral SNHL. 2 patients had mild to moderate bilateral SNHL with mean pure tone audiometry PTA of $42.5 \mathrm{dbHL}$ and 4 patients had moderate bilateral SNHL with mean PTA of $58.65 \mathrm{dbHL}$. One out of the 8 patients had unilateral auditory neuropathy spectrum disorder with PTA of 56.25 $\mathrm{dbHL}$ and one patient had unilateral mild SNHL with mean PTA of $38.25 \mathrm{dbHL}$. The mean degree of overall hearing loss of all patients

\section{9 assessed for} eligibility

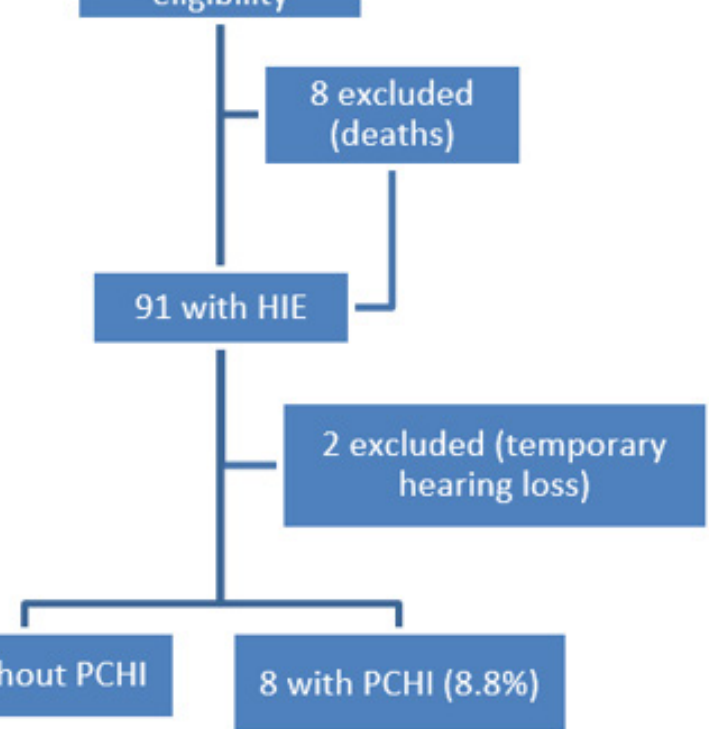

Figure 1: Flow chart diagram of patients' enrolment and number and percentage of patients with HIE and PCHI. 
Patients with SNHL underwent further aetiological investigation that were negative for cytomegalovirus in all cases and negative for genetics including Connexin 26 and A1555G mutation in bilateral cases. Neuroimaging including MRI IAMS did not show abnormalities apart from hypoxic ischaemic changes (Figures 2, 3).

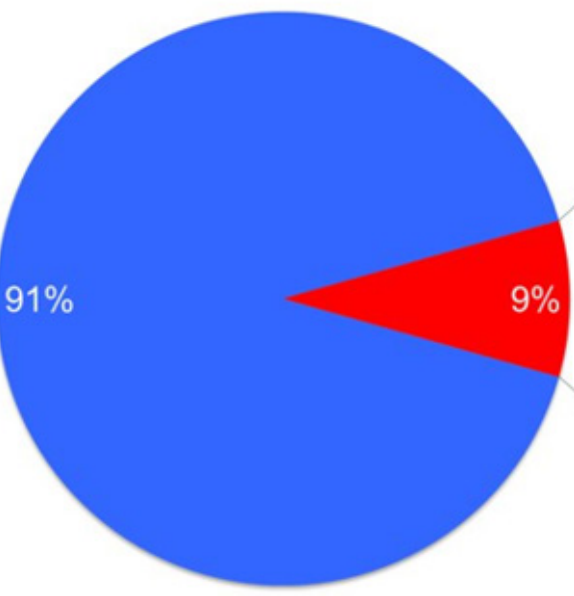

$7 \%$

- HIE without PCHI

Bilateral SNHL
Unilateral SNHL

Unilateral auditory neuropathy

Figure 2: Types of permanent childhood hearing impairment in HIE and its percentage.

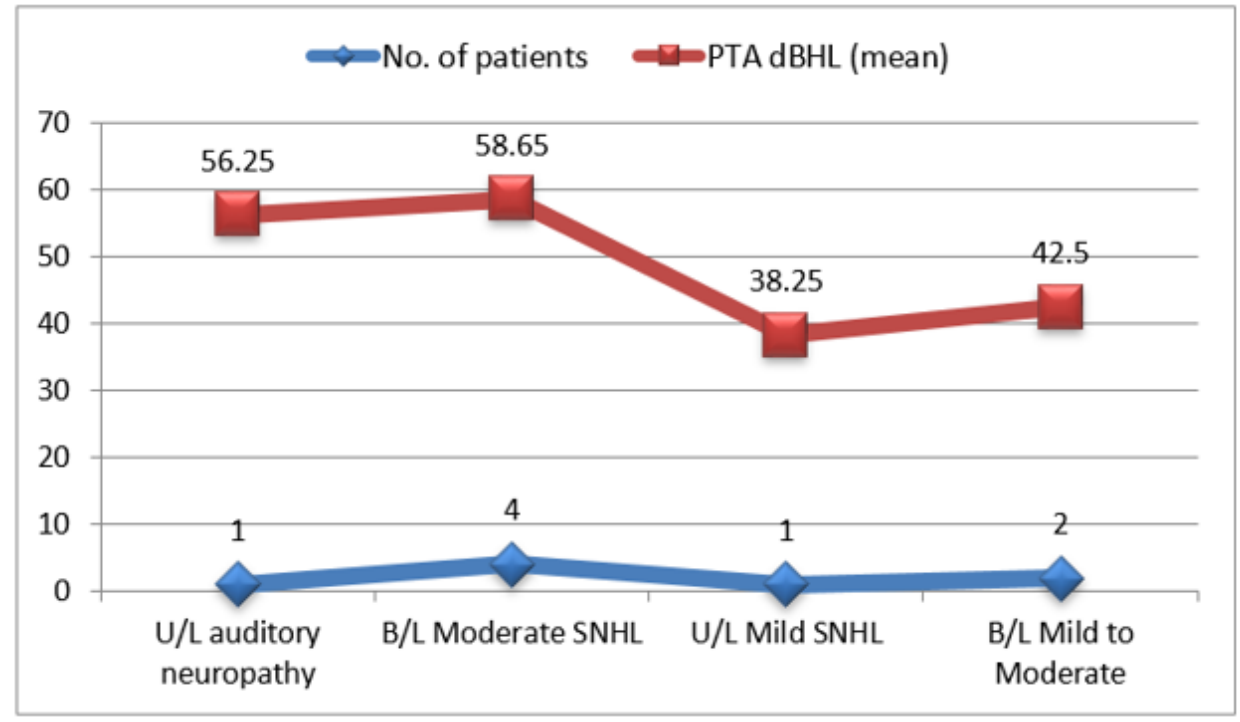

Figure 3: Diagram showing patients with hearing impairment and their average pure tone audiometry (U/L- Unilateral, B/L- Bilateral).

\section{Discussion}

In our cohort of mild, moderate and severe HIE, the incidence of PCHI is $8.8 \%$ which is similar to recent reported studies $[5,6]$. The mean degree of hearing loss was 51.4 ( \pm SD 14.4) dbHL which indicated a significant hearing loss which can affect speech and language development if there is no early intervention. Our results showed that there was no correlation between the severity of HIE and degree of hearing loss. However, there was a significant association of PCHI with infants who had low Apgar score at 1 and 5 minutes, PPHN and loop diuretics. Most important there was no correlation with the use of gentamicin which is in contrast to Smit, et al. [5] but similar to Fitzgerald, et al. study [6]. Smit, et al. [5] found an association of hearing loss with high gentamicin, low blood glucose and low ph. Fitzgerald, et al. [6] study demonstrated association between abnormal glucose in the first hour of delivery, low Apgars, need for resuscitation and raised creatinine and liver function in the initial days following birth.

Our results showed that there was a significant association of PCHI with infants who had low Apgar score at 1 and 5 minutes. Therefore, we hypothesise that acute perinatal hypoxia even with 
early recovery may be a cause for hearing loss and this data may help guide further research. Ribeiro, et al. [12] studied 181 term infants who passed newborn hearing screen using transient evoked otoacoustic emission test (TOAEs) into 3 groups. Group 1 (20) babies with asphyxia, Group 2 (111) babies with low Apgar score [ $<4$ at $1 \mathrm{~min} \&<6$ at $5 \mathrm{~min}$ ] and Group 3 (50 normal babies). They found on diagnostic OAE that Group 1 and 2 had lower signal to noise ratio (SNR) on TOAEs compared to Group 3. Also, increased latency of ABR waves I and III was noted in Group 1 babies. The study demonstrated that alterations occurred in both the cochlear and the neural components in the infants who had perinatal asphyxia of all grades [12].

We found significant association of permanent hearing loss with patient of HIE who had PPHN. Similar correlation was found in Hendricks-Munoz and Walton study, who found extremely high rate of SNHL in 21 of 40 infants who developed persistent foetal circulation. Duration of hyperventilation and ventilation correlated significantly with hearing loss in their study. Also, there was significant association of PCHI in those who received loop diuretics however the number of patients in our study is very small $(n=3 / 91)$ [13].

In our cohort of patients, we didn't find significant correlation of PCHI and the use aminoglycosides or anticonvulsants which is reassuring. However, in other studies such as Pouranian, et al. [14] which looked at the prevalence of hearing loss in newborns admitted to the neonatal intensive care, $19(14.8 \%)$ of the 105 cases who were administered antibiotics (aminoglycoside) had hearing loss, which was a significantly greater number than that observed in the babies who did not receive antibiotics $(\mathrm{P}=0.001)$ [14]..

\section{Limitations of the study}

This study was a single centre retrospective study which included small number of babies. Data on other systemic effects of HIE and long term neurodevelopmental outcomes were not looked at. In our study, we did not study any blood test results following birth on these babies and look at the correlation with hearing loss compared to other recent studies.

However, our study looked at the incidence of PCHI in all grades of HIE (mild, moderate and severe) and describes the degree and severity and types of permanent hearing loss. It involved detailed methodology of investigation of the permanent hearing loss and excluded other causes of hearing loss such as genetic and structural causes which can be some of the confounding factors.

\section{Conclusion}

Our study shows that babies with HIE who have persistent pulmonary hypertension, low Apgar scores at 1 and 5 minutes and those on loop diuretic medications are at increased risk for PCHI. Infants with mild HIE are also vulnerable to PCHI and it is important that they are not missed in the follow up. This data will allow further research with a larger cohort of babies to look at these risk factors and correlating with long-term neurodevelopmental outcome.

\section{Acknowledgement}

Parents and families of participated patients.

\section{Conflict of Interest}

No conflict of interest.

\section{Funding}

This research did not receive any specific grant from funding agencies in the public, commercial, or not-for-profit sectors.

\section{References}

1. Edwards AD, Gunn AJ, Halliday H, Juszczak E, Levene M, et al. (2010) Neurological outcomes at 18 months of age after moderate hypothermia for perinatal hypoxic ischaemic encephalopathy: synthesis and meta-analysis of trial data. BMJ 340: c363.

2. Eiserman WD, Hartel DM, Shisler L, Buhrmann J, White KR et al. (2008) Using otoacoustic emissions to screen for hearing loss in early childhood care settings. Int J Pediatr Otorhinolaryngol 72(4): 475-482.

3. Mwaniki MK, Atieno M, Lawn JE, Newton CRJC (2012) Long-term neurodevelopmental outcomes after intrauterine and neonatal insults: a systematic review. Lancet 379(9814): 445-452.

4. Jiang ZD, Wang J, Brosi DM, Shao XM, Wilkinson AR (2004) One-third of term babies after perinatal hypoxia-ischaemia have transient hearing impairment: dynamic change in hearing threshold during the neonatal period. Acta Paediatr 93(1): 82-87.

5. Smit E, Liu X, Gill H, Sabir H, Jary S, et al. (2013) Factors associated with permanent hearing impairment in infants treated with therapeutic hypothermia. J Pediatr 163: 995-1000.

6. Fitzgerald MP, Reynolds A, Garvey CM, Norman G, King MD, et al. (2019) Hearing impairment and hypoxia ischaemic encephalopathy: Incidence and associated factors. Eur J Paediatr Neurol 23(1): 81-86.

7. Gülsüm Kadıoğlu Șimșek, H Gözde Kanmaz Kutman, Fuat Emre Canpolat, Mehmet Büyüktiryaki, Yaprak Engin Üstün (2020) Hearing screening failure rate in newborn infants with hypoxic ischemic encephalopathy. Int J Pediatr Otorhinolaryngol 128: 109691.

8. https://www.gov.uk/publichealth/newbornhearingscreening.

9. (2013) Guidelines for the early audiological assessment and management of babies referred from the Newborn Hearing Screening Programme vs 3.1.

10. https://www.thebsa.org.uk/wp-content/uploads/2018/11/0D10432-Recommended-Procedure-Pure-Tone-Audiometry-August-2018FINAL.pdf

11. https://www.baap.org.uk/documents-guidelines-pathways-andclinical-standards.html

12. Ribeiro GE, da Silva DP, Montovani JC (2014) Avaliação dos níveis de resposta das emissões otoacústicas em neonatos com asfixia perinatal [Assessment of levels of otoacoustic emission response in neonates with perinatal asphyxia]. Rev Paul Pediatr 32(3): 189-193.

13. Hendricks-Munoz KD, Walton DP (1988) Hearing loss in infants with persistent fetal circulation. Pediatrics 81(5): 650-656.

14. Pourarian Sh, Khademi B , Pishva N, Jamali A (2012) Prevalence of hearing loss in newborns admitted to neonatal intensive care unit. Iran J Otorhinolaryngol 24(68): 129-134. 